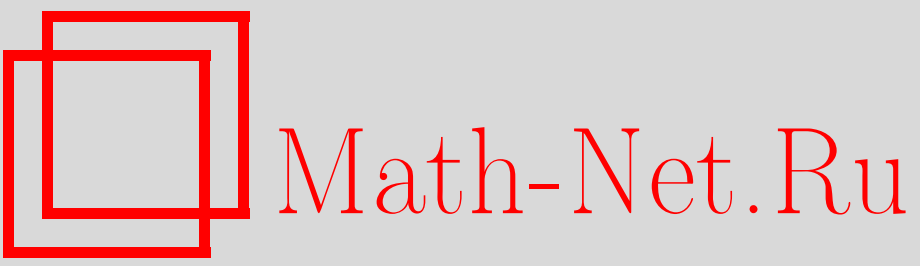

С. В. Яхонтов, Вычисление гипергеометрических рядов с квазилинейной временной и линейной ёмкостной сложностью, Вестн. Сам. гос. техн. ун-та. Сер. Физ.-мат. науки, 2011, выпуск 3(), 149-156

DOI: https://doi.org/10.14498/vsgtu924

Использование Общероссийского математического портала Math-Net.Ru подразумевает, что вы прочитали и согласны с пользовательским соглашением

http: //www. mathnet.ru/rus/agreement

Параметры загрузки:

IP : 34.227 .88 .159

26 апреля 2023 г., 09:29:50 


\title{
Информатика
}

\author{
УДК 519.677
}

\section{ВЫЧИСЛЕНИЕ ГИПЕРГЕОМЕТРИЧЕСКИХ РЯДОВ С КВАЗИЛИНЕЙНОЙ ВРЕМЕННОЙ И ЛИНЕЙНОЙ ЁМКОСТНОЙ СЛОЖНОСТЬЮ}

\section{С. В. Яхонтов}

Санкт-Петербургский государственный университет, математико-механический факультет, 198504, Санкт-Петербург, Старый Петергоф, Университетский пр-т, 28.

E-mails: sergey_home_mail@inbox.ru

\begin{abstract}
Проводится построение простого для практической реализачии алгоритма со сложностью $\mathrm{O}\left(M(n) \log (n)^{2}\right)$ по времени и $\mathrm{O}(n)$ по памяти для вычисления гипергеометрических рядов с рачиональными коэффичиентами на машине Шёнхаге, где $M(n)$ - сложность умножения целых чисел. Показывается, что данный алгоритм пригоден в практической информатике для построения конструктивных аналогов часто используемых констант математического анализа.
\end{abstract}

Ключевые слова: конструктивные вещественные числа, гипергеометрические ряды, квазилинейная временная сложность, линейная ёмкостная сложность.

Введение. Предлагается простой для практической реализации алгоритм, квазилинейный по времени и линейный по памяти, на машине Шёнхаге [1], для вычисления приближённых значений гипергеометрических рядов с рациональными коэффициентами. Данные ряды используются для расчёта некоторых констант математического анализа и элементарных функций в рациональных точках.

С помощью $S \operatorname{ch}(F Q L I N T I M E / / L I N S P A C E)$ будем обозначать класс алгоритмов, вычислимых на машине Шёнхаге, квазилинейных по времени и линейных по памяти. Основной особенностью машины Шёнхаге является возможность рекурсивных вызовов процедур. Под квазилинейностью понимается ограниченность сверху функцией вида $\mathrm{O}\left(n \log (n)^{k}\right)$ при некотором $k$.

Отличительной особенностью алгоритмов, основанных на разложениях в ряды, является относительная простота как самих алгоритмов, так и анализа их вычислительной сложности, так как при этом обычно производятся только арифметические операции над рациональными числами без обращения к дополнительным алгоритмам. Для вычислений с небольшим количеством знаков после двоичной точки ряды эффективнее других способов в связи с небольшими константами в оценках вычислительной сложности. Поэтому такие алгоритмы важны в информатике для практических приложений.

Известно [2], что линейно сходящиеся гипергеометрические ряды с рациональными коэффициентами можно вычислять с помощью метода двоичного деления с временной сложностью $\mathrm{O}\left(M(n)(\log (n))^{2}\right)$ и ёмкостной слож-

Яхонтов Сергей Викторович (к.ф.-м.н.), доцент, каф. информатики. 
ностью $\mathrm{O}(n \log (n))(M(n)$ - сложность умножения $n$-битовых целых чисел). В последнее время появились работы, например [3], в которых описываются алгоритмы (на основе модифицированного метода двоичного деления) вычисления значений линейно сходящихся гипергеометрических рядов с временной сложностью $\mathrm{O}\left(M(n)(\log (n))^{2}\right)$ и ёмкостной сложностью $\mathrm{O}(n)$. $\mathrm{B}[4]$ предложены простые для практической реализации алгоритмы для вычисления степенных рядов с полиномиальной временной и линейной ёмкостной сложностью.

Недостатком метода из [3] является относительная сложность практической реализации. В настоящей работе предлагается простой для практической реализации алгоритм, который, так же как и алгоритм из [3], является квазилинейным по времени и линейным по памяти. Идея работать с некоторой заданной точностью для того, чтобы вычислить значение гипергеометрического ряда с линейной ёмкостной сложностью, предложена в [5].

Вычислительная сложность конструктивных вещественных чисел и функций подробно освещается в монографии [6]. Множество конструктивных вещественных чисел с квазилинейной по времени и линейной по памяти сложностью вычисления двоично-рациональных приближений будем обозначать $S c h(F Q L I N T I M E / / L I N S P A C E)_{C F}$ (данное множество можно определить по аналогии с множеством FLINSPACE вычислимых действительных чисел [4] с учётом другой вычислительной модели).

Везде далее через $n$ будет обозначаться длина записи точности вычисления $2^{-n}$ двоично-рациональных приближений. Под функцией $\log (k)$ будем понимать логарифм по основанию 2.

1. Метод двоичного деления. Данный метод предназначен для вычисления значений линейно сходящихся рядов с рациональными коэффициентами, в частности, для вычисления гипергеометрических рядов вида

$$
S=\sum_{i=0}^{\infty} \frac{a(i)}{b(i)} \prod_{j=0}^{i} \frac{p(j)}{q(j)},
$$

где $a, b, p, q$-полиномы с целыми коэффициентами; данный ряд линейно сходится, если его частичная сумма

$$
S(\mu(k))=\sum_{i=0}^{\mu(k)} \frac{a(i)}{b(i)} \prod_{j=0}^{i} \frac{p(j)}{q(j)},
$$

где $\mu(k)$ - линейная функция от $k$, отличается от точного значения не более чем на $2^{-k}:|S-S(\mu(k))| \leqslant 2^{-k}$.

В классическом варианте метод двоичного деления состоит в следующем. Обозначим $k_{1}=\mu(k)$. Рассмотрим частичную сумму (2) для некоторых чисел $i_{1}$ и $i_{2}, 0 \leqslant i_{1} \leqslant k_{1}, 0 \leqslant i_{2} \leqslant k_{1}, i_{1} \leqslant i_{2}$ :

$$
S\left(i_{1}, i_{2}\right)=\sum_{i=i_{1}}^{i_{2}} \frac{a(i) p\left(i_{1}\right) \ldots p(i)}{b(i) q\left(i_{1}\right) \ldots q(i)} .
$$

Будем вычислять величины $P\left(i_{1}, i_{2}\right)=p\left(i_{1}\right) \ldots p\left(i_{2}\right), Q\left(i_{1}, i_{2}\right)=q\left(i_{1}\right) \ldots q\left(i_{2}\right)$, $B\left(i_{1}, i_{2}\right)=b\left(i_{1}\right) \ldots b\left(i_{2}\right)$ и $T\left(i_{1}, i_{2}\right)=B\left(i_{1}, i_{2}\right) Q\left(i_{1}, i_{2}\right) S\left(i_{1}, i_{2}\right)$. Если $i_{1}=i_{2}$, то 
величины вычисляются напрямую. Иначе ряд делится на две части, левую и правую, и величины $P\left(i_{1}, i_{2}\right), Q\left(i_{1}, i_{2}\right), B\left(i_{1}, i_{2}\right)$ вычисляются для каждой из частей рекурсивно. Затем полученные величины комбинируются:

$$
\begin{aligned}
& P\left(i_{1}, i_{2}\right)=P_{l} P_{r}, \quad Q\left(i_{1}, i_{2}\right)=Q_{l} Q_{r}, \quad B\left(i_{1}, i_{2}\right)=B_{l} B_{r}, \\
& T\left(i_{1}, i_{2}\right)=B_{r} Q_{r} T_{l}+B_{l} P_{l} T_{r} .
\end{aligned}
$$

Алгоритм начинает свою работу с $i_{1}=0, i_{2}=k_{1}$. После вычисления величин $T\left(0, k_{1}\right), B\left(0, k_{1}\right), Q\left(0, k_{1}\right)$ осуществляется деление $T\left(0, k_{1}\right)$ на $B\left(0, k_{1}\right) Q\left(0, k_{1}\right)$, чтобы получить результат с заданной точностью. Длины чисел $T\left(0, k_{1}\right)$ и $B\left(0, k_{1}\right) Q\left(0, k_{1}\right)$ пропорциональны $k \log (k)$, то есть алгоритм двоичного деления является квазилинейным по памяти; временная сложность данного алгоритма - $\mathrm{O}\left(M(k) \log (k)^{2}\right)[2]$.

2. Алгоритм вычисления гипергеометрических рядов из [3]. В алгоритме из [3] вычисляются редуцированные числитель и знаменатель дроби $\widehat{T} / \widehat{Q}$, где $\widehat{T}=T B Q, \widehat{Q}=B Q$, имеющие длину $\mathrm{O}(n)$, тогда как в классическом варианте вычисляются числитель и знаменатель длины $\mathrm{O}(n \log (n))$. Затем производится деление редуцированного числителя на редуцированный знаменатель с тем, чтобы получить значение суммы ряда с заданной точностью. Параметром усовершенствованного алгоритма из [3] является модуль $m-$ верхняя граница длины редуцированных $\widehat{T}$ и $\widehat{Q}$; модуль $m$ вычисляется как произведение достаточного числа простых чисел. Ряд разбивается на $N / G$ групп, где $G$ - величина, зависящая от $m$, и для каждой группы применяется классический алгоритм двоичного деления. Далее вычисленные значения комбинируются по модулю $m$ с использованием реконструкции рациональных чисел с тем, чтобы получить $\widehat{T}$ и $\widehat{Q}$.

Недостатком данного алгоритма является необходимость оценивать величину $m$ для каждого вида ряда отдельно; в [3] для этого предлагается достаточно трудоемкий метод, на примере которого оценивается $m$ для ряда $\zeta(3)$. Недостатком алгоритма из [3] является также необходимость реализовывать достаточно сложный алгоритм реконструкции рациональных чисел (rational number reconstruction), имеющий временную сложность $\mathrm{O}(M(n) \log (n))$ и ёмкостную сложность $\mathrm{O}(n)$.

3. Основной алгоритм класса $S \operatorname{ch}(F Q L I N T I M E / / L I N S P A C E)$. Модифицируем метод двоичного деления для гипергеометрических рядов так, чтобы алгоритм получился простой и при этом вычисления находились в пределах класса $S \operatorname{ch}(F Q L I N T I M E / / L I N S P A C E)$.

Пусть требуется вычислить значения гипергеометрического ряда (1) с точностью $2^{-n}$. Для этого достаточно вычислить частичную сумму (2) с точностью $2^{-(n+1)}$, так как

$$
\begin{aligned}
&\left|S-S(\mu(n+1))^{*}\right| \leqslant|S-S(\mu(n+1))|+\left|S(\mu(n+1))-S(\mu(n+1))^{*}\right| \leqslant \\
& \leqslant 2^{-(n+1)}+2^{-(n+1)}=2^{-n} .
\end{aligned}
$$

Здесь $S(\mu(n+1))^{*}$ - обозначение для приближенного значения $S(\mu(n+1))$. Обозначим $r=\mu(n+1)$. Возьмем минимальную величину $k_{1}$ такую, что $2^{k_{1}} \geqslant r$; пусть $r_{1}=\left\lceil r / k_{1}\right\rceil$. Запишем частичную сумму (2) в виде

$$
P(r)=\sigma_{1}+\tau_{2}\left[\sigma_{2}+\tau_{3}\left[\sigma_{3}+\ldots+\tau_{k_{1}-1}\left[\sigma_{k_{1}-1}+\tau_{k_{1}} \sigma_{k_{1}}\right]\right]\right],
$$




$$
\begin{aligned}
\text { где } \sigma_{1} & =\sum_{i=0}^{r_{1}-1} \frac{a(i)}{b(i)} \prod_{j=0}^{i} \frac{p(j)}{q(j)}=\sigma_{1}^{\prime}, \\
\tau_{2} & =\prod_{j=0}^{r_{1}} \frac{p(j)}{q(j)}, \quad \sigma_{2}=\frac{a\left(r_{1}\right)}{b\left(r_{1}\right)}+\sum_{i=r_{1}+1}^{2 r_{1}-1} \frac{a(i)}{b(i)} \prod_{j=r_{1}+1}^{i} \frac{p(j)}{q(j)}=\xi_{2}+\sigma_{2}^{\prime}, \\
\tau_{3} & =\prod_{j=r_{1}+1}^{2 r_{1}} \frac{p(j)}{q(j)}, \quad \sigma_{3}=\frac{a\left(2 r_{1}\right)}{b\left(2 r_{1}\right)}+\sum_{i=2 r_{1}+1}^{3 r_{1}-1} \frac{a(i)}{b(i)} \prod_{j=2 r_{1}+1}^{i} \frac{p(j)}{q(j)}=\xi_{3}+\sigma_{3}^{\prime}, \ldots
\end{aligned}
$$

Величины $\sigma_{t}^{\prime}$ будем вычислять классическим методом двоичного деления для суммы $(3)$, где $i_{1}=(t-1) r_{1}+1, i_{2}=t \cdot r_{1}-1$; для $\sigma_{1}^{\prime}$ возьмем $i_{1}=0, i_{2}=r_{1}-1$.

Введём следующие обозначения: $\omega=l(W)+1, W=\max (A, B)$, где $A=$ $=\max _{i=0 . . r}(|a(i)|,|b(i)|), B=\max _{j=0 . . r}(|p(j)|,|q(j)|), l(u)$ - длина битового представления $u$. Оформим оценку вычислительной сложности расчёта $\sigma_{t}$ и $\tau_{t}$ в виде двух лемм.

Лемма 1. Временная сложность алгоритма двоичного деления для вычисления $\sigma_{t}$ ограничена сверху $\mathrm{O}(M(r) \log (r))$; ёмкостная сложность ограничена сверху $\mathrm{O}(r)$.

До каз а тель ст в о. Рассмотрим произвольную максимальную цепочку рекурсивных вызовов, полученных в результате вычислений в соответствии с формулами (4). Условимся, что нумерация в цепочке начинается с самого глубокого элемента цепочки: $i=1, \ldots, \varsigma$, где $\varsigma$ - длина цепочки, $\varsigma \leqslant\left\lceil\log \left(2 r_{1}\right)\right\rceil$.

Пользуясь методом математической индукции по $i$, покажем, что длина представления числа $T$ на глубине $i$ при вычислении $\sigma_{t}^{\prime}$ удовлетворяет соотношению $l\left(T_{i}\right)<2^{i+1} \omega+2^{i}$. При этом заметим, что $l\left(P_{i}\right) \leqslant 2^{i} \omega, l\left(Q_{i}\right) \leqslant 2^{i} \omega$, $l\left(B_{i}\right) \leqslant 2^{i} \omega$, так как при увеличении $i$ происходит удвоение длины числа. База индукции: $i=1, l\left(T_{1}\right) \leqslant 2 \omega<2^{2} \omega+2^{1} ;$ индукционный переход:

$$
l\left(T_{i+1}\right)<2^{i} \omega+2^{i} \omega+2^{i+1} \omega+2^{i}+1<2^{(i+1)+1} \omega+2^{i+1} .
$$

Отметим, что исходя из этой оценки $T_{\varsigma}$ имеет длину $O(r)$, так как

$$
l\left(T_{\varsigma}\right)<C_{1} 2^{\varsigma} \omega \leqslant C_{2} \frac{r}{\log (r)} \log (r)=C_{2} r
$$

здесь учитывается тот факт, что все коэффициенты $a(i), b(i), p(j), q(j)$ являются полиномами.

Оценим временную сложность вычисления $\sigma_{t}^{\prime}$. При этом будем учитывать свойство функции сложности умножения: $2 M\left(2^{-1} m\right) \leqslant M(m)$ (квазилинейные и полиномиальные функции удовлетворяют этому свойству). Так как в узле дерева вызовов на уровне $i$ производится $C_{3}$ умножений $2^{\varsigma-i}$ чисел, длины которых не превосходят $2^{i+1} \omega+2^{i}$, получаем следующую оценку количества операций, требуемых для вычисления $\sigma_{t}^{\prime}$ :

$$
\operatorname{Time}\left(\sigma_{t}^{\prime}\right) \leqslant C_{3} \sum_{i=1}^{\varsigma} 2^{\varsigma-i} M\left(2^{i+1} \omega+2^{i}\right)<C_{4} \sum_{i=1}^{\varsigma} 2^{\varsigma-i} M\left(2^{i+2} \omega\right)=
$$




$$
\begin{aligned}
=C_{4} \sum_{i=1}^{\varsigma} 2^{\varsigma-i} M\left(2^{\varsigma-(\varsigma-(i+2))} \omega\right) \leqslant C_{5} \sum_{i=1}^{\varsigma} 2^{\varsigma-i} 2^{-\varsigma+(i+2)} M\left(2^{\varsigma} \omega\right) \leqslant \\
\leqslant C_{6} \varsigma M(r) \leqslant C_{7} \log (r) M(r)
\end{aligned}
$$

(здесь используется неравенство для $2^{\varsigma} \omega$ из оценки для $\left.l\left(T_{\varsigma}\right)\right)$. Заключительное деление дает $\mathrm{O}(M(r))$ операций.

Теперь оценим ёмкостную сложность вычисления $\sigma_{t}^{\prime}$. Учитывая, что в узле дерева вызовов вложенности $i$ количество памяти, расходуемой на временные переменные, - это $C_{8}\left(2^{i+1} \omega+2^{i}\right)$, получаем, что количество памяти во всех одновременно существующих рекурсивных вызовах оценивается следующим образом:

$$
\operatorname{Space}\left(\sigma_{t}^{\prime}\right) \leqslant \sum_{i=1}^{\varsigma} C_{8}\left(2^{i+1} \omega+2^{i}\right) \leqslant C_{9}\left(2^{\varsigma} \omega+2^{\varsigma}\right) \leqslant C_{10} r=\mathrm{O}(r) .
$$

Лемма 2. Временная сложность алгоритма двоичного деления для вычисления числителя и знаменателя $\tau_{t}$ ограничена сверху $\mathrm{O}(M(r) \log (r))$; ёмкостная сложность ограничена сверху $\mathrm{O}(r)$.

Доказательство. Оценки вычислительной сложности $\tau_{t}$ совпадают с таковыми для $\sigma_{t}$ в силу того, что неравенства из доказательства леммы 1 также актуальны и для $\tau_{t}$, если вычислять числитель и знаменатель $\sigma_{t}$ с помощью алгоритма двоичного деления для произведений.

Рассчитывать приближённые значения $P(r)^{*}$ с точностью $2^{-(n+1)}$ по формуле (5) будем в соответствии со следующим итеративным процессом:

$$
\begin{aligned}
& h_{1}(m)=\sigma_{k_{1}}^{*}, \\
& \widehat{h}_{i}(m)=\sigma_{k_{1}-i+1}^{*}+\tau_{k_{1}-i+2}^{*} h_{i-1}, \quad i=1, \ldots, k_{1}, \\
& h_{i}(m)=\widehat{h}_{i}(m)+\varepsilon_{i} ;
\end{aligned}
$$

при $i=k_{1}$ полагаем $P(r)^{*}=h_{k_{1}}(m)$. Здесь $m \geqslant r$ (величину $m$ выберем позже); $\sigma_{i}^{*}, \tau_{i}^{*}$ - приближения $\sigma_{i}, \tau_{i}$ с точностью $2^{-m}$. Величины $h_{i}(m)$ получаются отбрасыванием битов $q_{m+1} q_{m+2} \ldots q_{m+j}$ чисел $\widehat{h}_{i}(m)$ после двоичной точки, начиная с $(m+1)$-го, т. е.

$$
\left|\varepsilon_{i}\right|=\left|h_{i}(m)-\widehat{h}_{i}(m)\right|=0.0 \ldots 0 q_{m+1} q_{m+2} \ldots q_{m+j},
$$

а знак $\varepsilon_{i}$ совпадает со знаком $\widehat{h}_{i}(m)$ (ясно, что $\left.\left|\varepsilon_{i}\right|<2^{-m}\right)$.

Предположим, что выполняются следующие условия:

$$
|b(i)| \geqslant 2 \text { для всех } i, \quad|p(j)| /|q(j)| \leqslant 1 \text { для всех } j .
$$

Покажем, что тогда верны следующие две леммы.

Лемма 3. Для любого $i=1,2, \ldots, k_{1}$ справедлива оченка

$$
\left|h_{i}(m)\right|<(i+1) r_{1} W .
$$


Доказательство. Применим математическую индукцию по $j$ для $h_{j}(m)$. База индукции при $j$, равном $1:\left|h_{1}(m)\right| \leqslant r_{1} W+2^{-m}<2 r_{1} W$. Индукционный переход для $(j+1) \geqslant 2$ :

$$
\begin{aligned}
&\left|h_{j+1}(m)\right|=\left|\sigma_{k_{1}-(j+1)+1}^{*}+\tau_{k_{1}-(j+1)+2}^{*} h_{j}+\varepsilon_{j+1}\right| \leqslant \\
& \leqslant \frac{1}{2} r_{1} W+(j+1) r_{1} W+2^{-m}<((j+1)+1) r_{1} W .
\end{aligned}
$$

Лемма 4. Погрешность вычисления $h_{k_{1}}(m)$ по схеме (6) оценивается как

$$
\Delta\left(k_{1}, m\right)<2^{-m} m k_{1}^{2} W .
$$

Доказательство. Обозначим $H_{1}=\sigma_{k_{1}}, H_{i}=\sigma_{k_{1}-i+1}+\tau_{k_{1}-i+2} H_{i-1}$, $\eta(i, m)=\left|h_{i}(m)-H_{i}\right|$. Воспользуемся методом математической индукции для $\eta(j, m)$ по $j$. База индукции при $j$, равном 1 :

$$
\eta(1, m)=\left|h_{1}(m)-H_{1}\right|=\left|\sigma_{k_{1}}^{*}-\sigma_{k_{1}}\right|<2^{-m} .
$$

Индукционный переход для $(j+1) \geqslant 2$ :

$$
\begin{gathered}
\eta(j+1, m)=\mid \sigma_{k_{1}-(j+)+1}^{*}+\tau_{k_{1}-(j+1)+2}^{*} h_{j}(m)+\varepsilon_{j+1}- \\
\quad-\sigma_{k_{1}-(j+1)+1}-\tau_{k_{1}-(j+1)+2} H_{j} \mid< \\
<\left|\tau_{v}^{*} h_{j}(m)-\tau_{v} h_{j}(m)+\tau_{v} h_{j}(m)-\tau_{v} H_{j}\right|+2 \cdot 2^{-m} \leqslant \\
\quad \leqslant 2^{-m} h_{j}(m)+\eta(j, m)+2 \cdot 2^{-m} .
\end{gathered}
$$

Так как из $(9)\left|h_{j}(m)\right|<(j+1) r_{1} W$ и, по индукционному предположению, $\eta(j, m)<2^{-m} m j^{2} W$, можно записать

$$
\begin{aligned}
\eta(j+1, m)<2^{-m} & (j+1) r_{1} W+2^{-m} m j^{2} W+2 \cdot 2^{-m}< \\
& <2^{-m}(j+1) m W+2^{-m} m(j+1)^{2} W=2^{-m} m(j+2)^{2} W .
\end{aligned}
$$

Из $\Delta\left(k_{1}, m\right)=\eta\left(k_{1}, m\right)$ получаем искомое неравенство.

Из леммы 4 следует, что достаточно взять $m$ такое, чтобы выполнялось

$$
m \geqslant(n+1)+\lceil 2 \log (n+1)+2 \log (r)+\log (W)\rceil,
$$

для вычисления $P(r)^{*}$ с точностью $2^{-(n+1)}$.

Обозначим алгоритм расчёта гипергеометрического ряда, использующий схему (6), через LinSpaceBinSplit (linear space binary splitting).

АЛГОРИтм LinSpaceBinSplit. ПриБЛИЖёНное ЗНАчЕНИЕ РЯДА (1).

Вход: Запись точности вычисления $2^{-n}$.

Выход: Приближённое значение ряда (1) с точностью $2^{-n}$.

Описание:

1) вычисляем $r:=\mu(n+1)$; подбираем $k_{1}$ так, чтобы $2^{k_{1}} \geqslant r$; вычисляем $r_{1}:=\left\lceil r / k_{1}\right\rceil ;$ рассчитываем $W$

2) рассчитываем m по формуле (10); 
3) $h:=\sigma_{k_{1}}^{*}$ (с помощъю обычного алгоритма двоичного деления $c$ точноствю $\left.2^{-m}\right)$;

4) выполняем иикл по $i$ от 2 до $k_{1}$ :

а) рассчитываем $v_{1}:=\sigma_{k_{1}-i+1}^{*}$ с точностъю $2^{-m}$ с помощью объчного алгоритма двоичного деления и $v_{2}:=\tau_{k_{1}-i+2}^{*}$ с точностъю $2^{-m}$,

б) вычисляем выражение $\widehat{h}:=v_{1}+v_{2} h$,

в) $h$ присваиваем величину $\widehat{h}$, округлённую в соответствии с $(7)$;

5) на выход записываем $h$.

Оценим временную вычислительную сложность данного алгоритма, учитывая, что $r$ и $m$ линейно зависят от $n$ :

- $\mathrm{O}(\log (n))$ вычислений $\sigma_{t}$ дают $\mathrm{O}\left(M(n) \log (n)^{2}\right)$;

- $\mathrm{O}(\log (n))$ вычислений $\tau_{t}$ дают $\mathrm{O}\left(M(n) \log (n)^{2}\right)$;

- $\mathrm{O}(\log (n))$ умножений чисел длины $\mathrm{O}(n)$ дают $\mathrm{O}(M(n) \log (n))$;

итого получаем $\mathrm{O}\left(M(n) \log (n)^{2}\right)$ битовых операций. Ёмкостная сложность алгоритма LinSpaceBinSplit- $\mathrm{O}(n)$, так как во всех вычислениях в данном алгоритме фигурируют числа длины $\mathrm{O}(n)$.

Теорема. Модифицированный алгоритм двоичного деления для расчёта гипергеометрических рядов LinSpaceBinSplit принадлежит классу сложноcmu $\operatorname{Sch}(F Q L I N T I M E / / L I N S P A C E)$.

Заключение. В табл. 1, 2 приведены формулы и ряды для расчёта некоторых часто используемых на практике констант математического анализа. Данные ряды линейно сходятся (см. [2-4]), и они удовлетворяют условиям (8); следовательно, для расчёта их приближённых значений можно использовать алгоритм LinSpaceBinSplit, то есть перечисленные константы принадлежат множеству конструктивных чисел $S c h(F Q L I N T I M E / / L I N S P A C E)_{C F}$. Алгоритм LinSpaceBinSplit можно также использовать для вычисления приближений многих других констант и приближений элементарных функций в рациональных точках.

Если для умножения использовать алгоритм Шёнхаге-Штрассена с временной сложностью $\mathrm{O}(n \log (n) \log \log (n))$, то временная сложность алгоритма LinSpaceBinSplit будет О $\left(n \log (n)^{3} \log \log (n)\right)$; при использовании для умножения простого рекурсивного метода с временной сложностью $\mathrm{O}\left(n^{\log (3)}\right)$ временная сложность алгоритма LinSpaceBinSplit будет $\mathrm{O}\left(n^{\log (3)} \log (n)^{2}\right)$.

Отметим, что ряд константы $е$ сходится со скоростью $2^{-\mathrm{O}(n \log (n))}$, поэтому временная сложность расчёта $e$ в соответствии с алгоритмом LinSpaceBinSplit будет $\mathrm{O}(M(n) \log (n))$, а ёмкостная - $\mathrm{O}(n / \log (n))$.

Таблица 1

Формулы для расчёта констант

\begin{tabular}{|c|c|c|}
\hline Константы & Формулы & Ряды \\
\hline$e$ & $e=\exp (1)$ & $\exp (1)=2 \sum_{i=0}^{\infty} \frac{1}{2 i !}$ \\
\hline$\pi$ & $\pi=16 \alpha-4 \beta$ & $\begin{array}{c}\alpha=\operatorname{arctg}\left(\frac{1}{5}\right)=2 \frac{1}{5} \sum_{i=0}^{\infty}(-1)^{i} \frac{1}{2(2 i+1) 5^{2 i}} \\
\beta=\operatorname{arctg}\left(\frac{1}{239}\right)=2 \frac{1}{239} \sum_{i=0}^{\infty}(-1)^{i} \frac{1}{2(2 i+1) 239^{2 i}}\end{array}$ \\
\hline$\zeta(3)$ & & $\sum_{i=0}^{\infty} \frac{(-1)^{i}\left(205 i^{2}+250 i+77\right)((i+1) !)^{5}(i !)^{5}}{2((2 i+2) !)^{5}}$ \\
\hline
\end{tabular}


Ряды для расчёта констант

\begin{tabular}{c|c|c|c|c}
\hline Константы & $a(i)$ & $b(i)$ & $p(j)$ & $q(j)$ \\
\hline \multirow{2}{*}{$e$} & 1 & 2 & 1 & $j$ \\
$\pi$ & 1 & $2(2 i+1)$ & -1 & $5^{2}$ \\
\multirow{2}{*}{$\zeta(3)$} & 1 & $2(2 i+1)$ & -1 & $239^{2}$ \\
\hline
\end{tabular}

Из дальнейших исследований можно отметить построение алгоритмов расчёта гипергеометрических рядов с рациональными коэффициентами с временной сложностью $\mathrm{O}\left(n \log (n)^{k}\right), k \leqslant 3$, и при этом имеющих линейную ёмкостную вычислительную сложность.

\section{БИБЛИОГРАФИЧЕСКИЙ СПИСОК}

1. Schönhage A., Grotefeld A.F.W, Vetter E. Fast algorithms. A multitape Turing machine implementation. Mannheim, Germany: BI-Wissenschaftsverlag, 1994. 298 pp.

2. Haible B., Papanikolaou T. Fast multiprecision evaluation of series of rational numbers/ In: Algorithmic number theory: Proceedings of 3rd international symposium (Portland, OR, 1998) / Lect. Notes Comput. Sci., 1423. Berlin: Springer, 1998. Pp. 338-350.

3. Cheng H., Gergel B., Kim E., Zima E. Space-efficient evaluation of hypergeometric series // SIGSAM Bull., 2005. Vol. 39, no. 2. Pp. 41-52.

4. Yakhontov S. V. FLINSPACE constructive real numbers and functions (in Russian). Saarbrücken, Germany: Lambert Academic Publishing, 2010. 167 pp.

5. Gourdon X., Sebah P. Binary splitting method: http://numbers.computation.free.fr/ Constants/Algorithms/splitting.ps.

6. Ko K.-I Complexity theory of real functions. Progress in Theoretical Computer Science. Boston, MA: Birkhäuser Boston, Inc., 1991. 310 pp.

Поступила в редакцию 01/II/2011;

в окончательном варианте - 24/VIII/2011.

MSC: 03F60; 68Q17

\section{CALCULATION OF HYPERGEOMETRIC SERIES WITH QUASI-LINEAR TIME AND LINEAR SPACE COMPLEXITY}

\section{S. V. Yakhontov}

St. Petersburg State University, Mathematics and Mechanics Faculty, 28, Universitetskiy prosp., Stariy Petergoff, St. Petersburg, 198504.

E-mails: sergey_home_mail@inbox.ru

A simple for practical implementation algorithm with the time complexity $\mathrm{O}\left(M(n) \log (n)^{2}\right)$ and space complexity $\mathrm{O}(n)$ for the evaluation of hypergeometric series with rational coefficients on the Schönhage machine is constructed (here $M(n)$ is the complexity of integer multiplication). It is shown that this algorithm is suitable in practical informatics for constructive analogues of often used constants of analysis.

Key words: constructive real numbers, hypergeometric series, quasi-linear time, linear space complexity.

Original article submitted 01/II/2011; revision submitted $24 / \mathrm{VIII} / 2011$.

Sergey V. Yakhontov (Ph. D. (Phys. \& Math.)), Associate Professor, Dept. of Informatics. 